\title{
Voltage limitation analysis in strain-balanced InAs/GaAsN quantum dot solar cells applied to the intermediate band concept
}

P. G. Linares*a ${ }^{a}$, E. López ${ }^{a}$, I. Ramiro ${ }^{a}$, A. Datas ${ }^{a}$, E. Antolín ${ }^{a}$, Y. Shoji $i^{b}$, T. Sogabe ${ }^{b}$, Y. Okada ${ }^{b}$, A. Martí ${ }^{a}$, and A. Luque ${ }^{a}$

anstituto de Energía Solar, Universidad Politécnica de Madrid, 28040 Madrid, Spain,

${ }^{b}$ Research Center for Advanced Science and Technology, The University of Tokyo, 4-6-1

Komaba, Meguro-ku, Tokyo 153-8904, Japan

\begin{abstract}
Several attempts have been carried out to manufacture intermediate band solar cells (IBSC) by means of quantum dot (QD) superlattices. This novel photovoltaic concept allows the collection of a wider range of the sunlight spectrum in order to provide higher cell photocurrent while maintaining the open-circuit voltage $\left(V_{\mathrm{OC}}\right)$ of the cell. In this work, we analyze InAs/GaAsN QDIBSCs. In these cells, the dilute nitrogen in the barrier plays an important role for the strain-balance (SB) of the QD layer region that would otherwise create dislocations under the effect of the accumulated strain. The introduction of GaAsN SB layers allows increasing the light absorption in the QD region by multi-stacking more than 100 QD layers. The photo-generated current density $\left(J_{\mathrm{L}}\right)$ versus $V_{\mathrm{OC}}$ was measured under varied concentrated light intensity and temperature. We found that the $V_{\mathrm{OC}}$ of the cell at $20 \mathrm{~K}$ is limited by the bandgap of the GaAsN barriers, which has important consequences regarding IBSC bandgap engineering that are also discussed in this work.
\end{abstract}

Keywords

Solar cell, intermediate band, quantum dot, concentrated light, band anticrossing, GaAsN

*Corresponding author: p.garcia-linares@ies-def.upm.es 


\section{Introduction}

The intermediate band solar cell (IBSC) concept has been proposed as a means to achieve high efficiency photovoltaics $[1,2]$. The idea behind the concept relies on the use of an engineered material, known as intermediate band (IB) material, which hosts a collection of energy levels (the so-called IB) within the bandgap, i.e. between the valence band (VB) and the conduction band (CB). In an IBSC, the IB material has to be embedded between p- and n-emitters in order to isolate the IB levels from the contacts. A sketch of the energy band diagram of such IBSC is shown in Figure 1. With respect to its operation, the IBSC has to fulfill two essential requirements: on the one hand, the generation of an extra current by means of the exploitation of sub-bandgap photons in a two-step absorption processes through the IB and, on the other hand, the preservation of the output voltage with respect to the material without IB. This second requirement can also be regarded as if the solar cell $V_{\mathrm{OC}}$ is exclusively limited by its fundamental bandgap $\left(E_{\mathrm{G}}\right)$ and not by any of the two sub-bandgaps, neither $E_{\mathrm{H}}$ nor $E_{\mathrm{L}}$ (see Figure 1). Not fulfilling this voltage preservation would imply that the Shockley-Queisser efficiency limit cannot be surpassed and such material should be rejected as IB material candidate. Both requirements have already been experimentally verified in quantum dots (QD) IBSCs. In this respect, sub-bandgap photocurrent $\left(I_{\mathrm{L}}\right)$ originated by two-step photon absorption has been reported in $[3,4]$ while open-circuit voltage $\left(V_{\mathrm{OC}}\right)$ not limited by any of the IB material sub-bandgaps has been reported in [5-7] at low temperature. In order to accomplish these principles, the IBSC concept demands some requirements such as, for example, that the IB is partially filled with electrons during operation but their discussion is out of the scope of this work $[8,9]$.

\section{InAs/GaAsN quantum dot (QD) strain balance solar cells}

One method for making IB materials is by stacking QD layers. QDs confine electrons in the three spatial directions causing the appearance of discrete energy levels that act as IB. InAs/GaAs QD technology has been widely used to implement IBSCs and has been the first one that has allowed verifying the two aforementioned operation principles. However, these self-assembled QDs provide 
weak sub-bandgap absorption and, subsequently low additional $I_{\mathrm{L}}$. Several strategies have been proposed to counteract this lack of absorption, such as reducing the size of the dots [10], using high-index substrates to order and pack the in-plane dots [11] or employing metal nanoparticles [12] or diffraction grid patterns [13] as light trapping techniques. However, these techniques are still challenging to implement.

A straightforward approach to boost light absorption is to increase the number of QD layers. However, the practical implementation of multiple InAs/GaAs QD layers faces problems derived from the accumulation of strain. This strain is primarily caused by the large difference in lattice constant $\left(a_{\mathrm{lc}}\right)$ between the barrier material, GaAs, and the QD material, InAs. Strain is transmitted vertically in the growth direction so that it increases throughout the successive QD layers until it is inelastically relaxed causing the formation of dislocations in the crystal structure [14]. Therefore, the maximum number of stacked QD layers that can be grown becomes limited unless strain balance (SB) is used. This consists of the use of a tensile-strained barrier material, characterized by a lattice constant $a_{\mathrm{lc}}$ smaller than that of the substrate. These tensile-strained barriers compensate the compressive strain of the QD layers leading to a zero overall in-plane stress $[15,16]$. The successive compressive-tensile strain components lead to a strain symmetrization where no strain is vertically accumulated. A simplified sketch explaining the SB technique applied to a InAs/GaAs QD stack is shown in Figure 2. Horizontal arrows indicate the compressive and tensile strains exerted in each layer. GaN and $\mathrm{GaP}$ are suitable strain-balancing materials for the InAs/GaAs QD system since in both cases their lattice constant is smaller than that of the GaAs. Therefore, both $\operatorname{GaAs}_{1-\mathrm{x}} \mathrm{N}_{\mathrm{x}}$ or $\mathrm{GaAs}_{1-\mathrm{x}} \mathrm{P}_{\mathrm{x}}$ alloys can be chosen as barrier materials for strain balancing. The first of them is studied in this work.

\section{Experiments}

InAs/ $\mathrm{GaAs}_{1-\mathrm{x}} \mathrm{N}_{\mathrm{x}}$ QD cells were fabricated at the University of Tokyo with $x=0.01$ following the typical layer structure of an IBSC, i.e. sandwiching the stacked QD layers between $p$ - and $n$ - bulk emitters in order to isolate the intermediate levels from the contacts. Up to 25 InAs QD layers were grown using $20 \mathrm{~nm}$ thick intrinsic $\mathrm{GaAs}_{0.99} \mathrm{~N}_{0.01}$ spacers as $\mathrm{SB}$ material. Doping of the barrier or 
directly in the dots is commonly carried out in order to half-fill the IB with electrons $[2,4]$ and maximize below bandgap two photon absorption. However, in order to progress step by step with the study of this system and proceed with the study of voltage preservation first, QDs were intentionally undoped since doping is expected to have no impact on voltage preservation and the structure is simplified.

A GaAs control cell where the QD layers and the GaAsN spacers have been substituted by an intrinsic GaAs region was also prepared. Both QD and reference cells were grown on top of $001 n^{+}$ GaAs substrates, including a $1,000 \mathrm{~nm}$ thick $n$-base, a $250 \mathrm{~nm}$ thick $p$-emitter, a $30 \mathrm{~nm}$ thick AlGaAs window layer and a highly doped contact layer. Neither anti-reflecting coating (ARC) nor back surface field (BSF) layers were included for simplicity. Both solar cells were metalized with a dense metal grid pattern that should allow low series resistance $\left(r_{\mathrm{s}}\right)$ for high current transport at low power loss. A sketch of the layer structure of the QD cell is represented in Figure 3.

As stated by the band anticrossing (BAC) model [17,18], some nitride dilute alloys abruptly split their CB in two bands as small amounts of nitrogen are incorporated. The energy edge of these bands, $E_{ \pm}(\mathrm{k})$, is described by

[17], where $E^{\mathrm{N}}$ is the position of the isoelectronic impurity $(\mathrm{N})$ within the host compound, $V_{\text {int }}$ is the interaction potential between the two bands, $x$ is the variable representing the nitrogen content and $E^{\mathrm{C}}(\mathrm{k})$ is the $\mathrm{CB}$ dispersion of the non-nitrogen semiconductor. In our case, $x=0.01$ implies a shrinkage of the GaAs$s_{1-x} \mathrm{~N}_{\mathrm{x}}$ bandgap, when compared to GaAs, of $\sim 200 \mathrm{meV}$. In fact, also the IBSC structure proposed in Ref. [19] relies on the BAC effect splitting the CB of the host material in two ( $E_{\text {- }}$ and $E_{+}$with $E_{+}$playing the role of the $\mathrm{CB}$ ). This split is believed to create a three-band configuration in GaAsN that allows collecting sub-bandgap energy photons through the $\mathrm{VB} \rightarrow E$. $\left(E_{\mathrm{H}}\right)$ and $E_{-} \rightarrow E_{+}\left(E_{\mathrm{L}}\right)$ transitions. The viability of this IBSC structure will be later discussed in connection to the results obtained in this work.

Regarding the QD approach, an approximate band diagram structure, assuming the dots located in a flatband region and including the QD layers and the $\mathrm{GaAs}_{0.99} \mathrm{~N}_{0.01}$ SB spacers, is shown in Figure 4a where the rest of materials used in the device structure are also indicated. Double arrows and 
bracketed numbers represent the transitions implied in the operation of this QD solar cell. The corresponding fundamental wavelengths at which these transitions occur are given as well. All these transitions can be correlated with the external quantum efficiency (QE) shown in Figure 4b. QEs have been normalized for making their comparison easier. The QE plot includes also a QDIBSC solar cell where the strain-balancing strategy has been substituted by thicker GaAs spacers that absorb most of the strain and also allow stacking a large number of QD layers (25 in this case). Each of the four transitions plotted in the previous band diagram can be assigned to a peak of the QE which corresponds to: (1) the electronic transitions through the QDs or $E_{\mathrm{H}}$; (2) transitions through the GaAsN bandgap (not present in the InAs/GaAs QD sample); (3) transitions through the wetting layer (WL), i.e. the quantum well (QW) associated to the QDs and always attached to them when grown by means of the Stranski-Krastanov growth mode [20]; and (4) transitions through the GaAs bandgap.

\section{Results and discussion}

The InAs/GaAsN and the GaAs control cell were subjected to characterization using concentrated light. The measurement of the photo-generated current density $\left(J_{\mathrm{L}}\right)$ as a function of the $V_{\mathrm{OC}}$ for different light concentrations constitute a useful tool for the evaluation of the voltage preservation in IBSCs [6,7]. For a detailed description of this technique, the reader is encouraged to visit Ref. [21]. It can be shown that the $J_{\mathrm{L}}-V_{\mathrm{OC}}$ curve equals the dark current curve of the solar cell without the influence of the $r_{\mathrm{S}}$. At low voltage, an ideal IBSC exhibits larger radiative recombination than a reference cell without IB due to the additional radiative transitions that take place through the intermediate levels. However, when voltage preservation occurs, the IBSC theory predicts [22] that both IBSC and reference cell dark curves converge at high voltage. In practice, however, such convergence cannot be verified by means of the direct measurement of the dark curve of the cells due to the impact of series resistance but by comparing the aforementioned $J_{\mathrm{L}}-V_{\mathrm{OC}}$ characteristic of both curves. The reason for this, as mentioned, is that $J_{\mathrm{L}}-V_{O C}$ plots are not affected by the series resistance of the device. These measurements can also be carried at low temperature in order to determine the highest limit for the maximum $V_{\mathrm{OC}}$ that can be achieved by the cell and find out 
whether any sub-bandgap limits the performance of the IBSC. When concentrated illumination is increased and any of these sub-bandgaps limits the performance of the cell, the $V_{\mathrm{OC}}$ saturates at values corresponding to the sub-bandgap energy divided by the electron charge, $E_{\mathrm{L}} / q$ or $E_{\mathrm{H}} / q$, instead of $E_{\mathrm{G}} / q$ ( $q$ being the electron charge). Figure 5 shows $J_{\mathrm{L}}-V_{\mathrm{OC}}$ plots of the InAs/GaAsN QD cell measured up to $\sim 500$ suns and at different temperatures. The corresponding dark $J-V$ curves are also shown as a reference. The superposition principle (which we say is fulfilled when both dark $J$ $V$ curve and $J_{\mathrm{L}}-V_{\mathrm{OC}}$ concentrated light curves match) is not fully preserved as temperature $(T)$ is lowered further what is likely caused by a high series resistance. Actually, at $T=150 \mathrm{~K}$, both curves do not match at all, although they remain parallel one to each other. At lower temperatures, the dark curves are far away from the corresponding concentrated light $J_{\mathrm{L}}-V_{\mathrm{OC}}$ curves.

The $J_{\mathrm{L}}-V_{\mathrm{OC}}$ plots of the QD-IBSC containing $\mathrm{GaAs}_{0.99} \mathrm{~N}_{0.01}$ shift towards higher voltages as $T$ decreases until they saturate at approximately $1.22 \mathrm{eV}(\lambda \sim 1020 \mathrm{~nm})$ for $T=20 \mathrm{~K}$ which is a value slightly lower than the bandgap of $\mathrm{GaAs}_{0.99} \mathrm{~N}_{0.01}$ at such a low temperature (estimated to be $1.29 \mathrm{eV}$ for these samples [18]). On the contrary, the InAs/GaAs QD cell and the GaAs control cell, with the structure shown in Fig. $4 \mathrm{~b}$, reach a $V_{\mathrm{OC}}$ close to the total bandgap $E_{\mathrm{G}}, 1.5 \mathrm{~V}$ (not shown in the plots in Fig. 5 but listed in Table I) at the same low temperature (20K) and under $\sim 500$ suns of concentrated light (in agreement with the results in ref. [6]). These results (corresponding to structures without $\mathrm{N}$ ) match therefore the expected GaAs bandgap at low $T$. Results reveal that $\mathrm{SB}$ InAs/GaAsN-based QD cells exhibit a maximum $V_{\mathrm{OC}}$ limited by their barrier material and not by the higher bandgap of the GaAs emitters making this system non ideal for 1-junction IBSC, where the optimum fundamental bandgap, $E_{\mathrm{G}}$, has to be close to $2 \mathrm{eV}$. It is noteworthy that the implementation of GaAsN-based IBSC would appropriately fit in multijunction designs [23], where lower bandgap materials are required for the bottom cell.

\section{Conclusions}

Regarding the implementation of QD-IBSCs, InAs/GaAsN has been proposed as a QD/barrier material that allows increasing the number of stacked QD layers and, therefore, has the potential to 
enhance sub-bandgap absorption. In this work, electro-optical characterization of QD-IBSCs based on InAs/GaAsN has been performed, including concentrated light $J_{\mathrm{L}}-V_{\mathrm{OC}}$ experiments, leading to the conclusion that such QD cell design does not fulfill the voltage preservation principle. Nevertheless, even if the introduction of nitrogen in the barriers of an InAs/GaAs QD cell reduces its potential as IBSC (because of a less favorable bandgap combination is obtained), other alternatives can be envisaged, such as substituting $\mathrm{N}$ by $\mathrm{P}[16]\left(\mathrm{GaAs}_{1-\mathrm{x}} \mathrm{P}_{\mathrm{x}}\right.$ bandgap is always larger than GaAs) in order to obtain an appropriate SB material, thickening the spacer layers so that the excess of strain is diluted [24] or using other QD material combinations based on semiconductors with higher $E_{\mathrm{G}}$ and/or higher $E_{\mathrm{L}} / E_{\mathrm{G}}$ ratio $[25,26]$ (e.g. using on $\mathrm{Al}_{\mathrm{x}} \mathrm{Ga}_{1-\mathrm{x}} \mathrm{As}$ as barrier material).

Three additional conclusions can also be inferred from our results:

1) The GaAsN dilute alloy might not be a suitable IB candidate bulk material for implementing an IBSC (contrary to what is proposed in Ref. [19]) if the band properties exhibited when inserted in the QD structures shown in this paper (not null density of states between the IB and the CB) prevail in bulk structures. More recent calculations of the density of states between the IB and the CB of GaAsN are in line with this interpretation [27]. In our results, the energy difference between its VB and the lower energy $\mathrm{CB}$, $\mathrm{E}_{-}$(and not $\mathrm{E}_{+}$), limits the quasi-Fermi Level (QFL) split and restricts the output voltage of the cell to values equal or lower than $E_{\mathrm{H}} / q$, where q is the electron charge. Actually, an appropriate IBSC made from GaAsN should have a $V_{\mathrm{OC}}$ only limited by the difference between its VB and the higher energy $\mathrm{CB}, E_{+}$.

2) The original motivation for implementing a multiple layer InAs/GaAs QD-IBSC including 1\% of $\mathrm{N}$ in the barriers, consisting on forming a SB material in order to allow an increased number of stacked QD layers to be grown, is clearly limited by the impossibility of recovering the voltage of the GaAs emitters. Actually, the InAs/GaAsN cell shows a $V_{\mathrm{OC}}$ limited by the $\mathrm{VB} \rightarrow E$. bandgap of the GaAsN and thus, nearly $300 \mathrm{mV}$ lower than the InAs/GaAs QD-IBSC. Moreover, the comparison of the SB QD structure with its reference counterpart (a cell with GaAs emitters and 
without IB material) reveals an unaccomplished voltage recovery as it is defined in Ref. [6], i.e.

$V_{\mathrm{OC}}$ of the QD cell noticeably lower than that of the reference cell.

3) Experimental evidence is provided on the fact that the $V_{\mathrm{OC}}$ of an IBSC with higher bandgap emitters (layers) sandwiching a lower bandgap IB material is limited by this last one, even under very high concentration and low temperatures.

\section{ACKNOWLEDGEMENTS}

This work has been supported by the European Commission through the Project NGCPV (Grant Number 283798). EL acknowledges the "Ayudas para la Formación de Personal Investigador (FPI) of the Spanish Government (EEBB-BES-2010-037659).

\section{REFERENCES}

[1] A. Luque and A. Martí, "Increasing the efficiency of ideal solar cells by photon induced transitions at intermediate levels," Physical Review Letters, vol. 78, pp. 5014-5017, 1997.

[2] A. Martí, L. Cuadra, and A. Luque, "Intermediate-band solar cells," in NEXT GENERATION PHOTOVOLTAICS: High Efficiency through Full Spectrum Utilization, A. Martí and A.Luque, Eds. Bristol: Institute of Physics Publishing, 2003, pp. 140-164.

[3] A. Martí, E. Antolín, C. R. Stanley, C. D. Farmer, N. López, P. Díaz, E. Cánovas, P. G. Linares, and A. Luque, "Production of Photocurrent due to Intermediate-to-Conduction-Band Transitions: A Demonstration of a Key Operating Principle of the Intermediate-Band Solar Cell," Physical Review Letters, vol. 97, p. 247701, 2006.

[4] Y. Okada, T. Morioka, K. Yoshida, R. Oshima, Y. Shoji, T. Inoue, and T. Kita, "Increase in photocurrent by optical transitions via intermediate quantum states in direct-doped InAs/GaNAs strain-compensated quantum dot solar cell," Journal of Applied Physics, vol. 109, pp. 024301-024301-5, 2011.

[5] E. Antolín, A. Martí, P. G. Linares, I. Ramiro, E. Hernández, C. D. Farmer, C. R. Stanley, and A. Luque, "Advances in quantum dot intermediate band solar cells," in 35th IEEE Photovoltaic Specialists Conference (PVSC), Honolulu, Hawaii, USA, 2010, p. 000065.

[6] P. G. Linares, A. Martí, E. Antolín, C. D. Farmer, I. Ramiro, C. R. Stanley, and A. Luque, "Voltage recovery in intermediate band solar cells," Solar Energy Materials and Solar Cells, vol. 98, pp. 240-244, 2012.

[7] P. G. Linares, A. Martí, E. Antolín, I. Ramiro, E. López, E. Hernández, D. Fuertes Marrón, I. Artacho, I. Tobías, P. Gérard, C. Chaix, R. P. Campion, C. T. Foxon, C. R. Stanley, S. I. Molina, and A. Luque, "Extreme voltage recovery in GaAs:Ti intermediate band solar cells," Solar Energy Materials and Solar Cells, vol. 108, pp. 175-179, 2013.

[8] A. Luque and A. Martí, "On the Partial Filling of the Intermediate Band in IB Solar Cells," Electron Devices, IEEE Transactions on, vol. 57, pp. 1201-1207, 2010.

[9] A. Martí, L. Cuadra, and A. Luque, "Partial filling of a quantum dot intermediate band for solar cells," IEEE Transactions on Electron Devices, vol. 48, pp. 2394-2399, 2001.

[10] A. Luque, A. Martí, E. Antolín, and P. G. Linares, "Intraband absorption for normal illumination in quantum dot intermediate band solar cells," Solar Energy Materials and Solar Cells, vol. 94, pp. 2032-2035, 2010. 
[11] K. Akahane, T. Kawamura, K. Okino, H. Koyama, S. Lan, Y. Okada, M. Kawabe, and M. Tosa, "Highly packed InGaAs quantum dots on GaAs(311)B," Applied Physic Letters, vol. 73, pp. 3411-3413, 1998.

[12] M. J. Mendes, A. Luque, I. Tobías, and A. Martí, "Plasmonic light enhancement in the nearfield of metallic nanospheroids for application in intermediate band solar cells," Applied Physics Letters, vol. 95, pp. 071105-071105-3, 2009.

[13] A. Mellor, I. Tobías, A. Martí, M. J. Mendes, and A. Luque, "Upper limits to absorption enhancement in thick solar cells using diffraction gratings," Progress in Photovoltaics: Research and Applications, vol. 19, pp. 676-687, 2011.

[14] A. Martí, N. López, E. Antolín, E. Cánovas, A. Luque, C. R. Stanley, C. D. Farmer, and P. Díaz, "Emitter degradation in quantum dot intermediate band solar cells," Applied Physics Letters, vol. 90, p. 233510, 2007.

[15] R. Oshima, A. Takata, and Y. Okada, "Strain-compensated InAs/GaNAs quantum dots for use in high-efficiency solar cells," Applied Physics Letters, vol. 93, p. 083111, 2008.

[16] V. Popescu, G. I. Bester, M. C. Hanna, A. G. Norman, and A. Zunger, "Theoretical and experimental examination of the intermediate-band concept for strain-balanced (In,Ga)As/Ga(As,P) quantum dot solar cells," Physical Review B, vol. 78, p. 205321, 2008.

[17] W. Shan, W. Walukiewicz, J. W. Ager, E. E. Haller, J. F. Geisz, D. J. Friedman, J. M. Olson, and S. R. Kurtz, "Band Anticrossing in GalnNAs Alloys," Physical Review Letters, vol. 82, p. $1221,1999$.

[18] J. Wu, W. Shan, and W. Walukiewicz, "Band anticrossing in highly mismatched III-V semiconductor alloys," Semiconductor Science and Technology, vol. 17, p. 860, 2002.

[19] N. López, L. A. Reichertz, K. M. Yu, K. Campman, and W. Walukiewicz, "Engineering the electronic band structure for multiband solar cells, Physical Review Letters, vol. 106, p. 028701, 2011.

[20] M. Sugawara, Self-assembled InGaAs/GaAs quantum dots vol. 60: Academic Press, 1999.

[21] P. G. Linares, A. Martí, E. Antolín, I. Ramiro, E. López, C. D. Farmer, C. R. Stanley, and A. Luque, "Low-Temperature Concentrated Light Characterization Applied to Intermediate Band Solar Cells," Photovoltaics, IEEE Journal of, vol. 3, pp. 753-761, 2013.

[22] A. Martí, E. Antolín, E. Cánovas, N. López, P. G. Linares, A. Luque, C. R. Stanley, and C. D. Farmer, "Elements of the design and analysis of quantum-dot intermediate band solar cells," Thin Solid Films, vol. 516, pp. 6716-6722, 2008.

[23] E. Antolín, A. Martí, P. G. Linares, I. Ramiro, E. Hernández, and A. Luque, "Raising the efficiency limit of the GaAs-based intermediate band solar cell through the implementation of a monolithic tandem with an AlGaAs top cell", in $25^{\text {th }}$ European Photovoltaic Solar Energy Conference (PVSEC), Valencia, Spain, 2010, p. 65.

[24] E. Antolín, A. Martí, C. D. Farmer, P. G. Linares, E. Hernández, A. M. Sánchez, T. Ben, S. I. Molina, C. R. Stanley, and A. Luque, "Reducing carrier escape in the InAs/GaAs quantum dot intermediate band solar cell," Journal of Applied Physics, vol. 108, p. 064513, 2010.

[25] P. G. Linares, A. Martí, E. Antolín, and A. Luque, "III-V compound semiconductor screening for implementing quantum dot intermediate band solar cells," Journal of Applied Physics, vol. 109, p. 014313, 2011.

[26] A. Luque, P. G. Linares, A. Mellor, V. Andreev, and A. Martí, "Some advantages of intermediate band solar cells based on type II quantum dots," Appl. Phys. Lett., vol. 103, 2013.

[27] R. Kudrawiec, A.V Luce, M. Gladysiewicz, M. Ting, Y.J Kuang, C.W Tu, O.D Dubon, K.M Yu, and W. Walukiewicz, "Electronic Band Structure of GaNPAs Highly Mismatched Alloys: Suitability for Intermediate-Band Solar Cells," Phys. Rev. Appl., vol 1, p. 034007, 2014. 


\begin{tabular}{|c|c|c|c|c|}
\hline \multicolumn{5}{|l|}{ Table I. Maximum $V_{\mathrm{OC}}$ values under concentrated light for different QD and reference cells } \\
\hline Cell structure / $T(\mathrm{~K})$ & $T=298 \mathrm{~K}$ & $T=150 \mathrm{~K}$ & $T=77 \mathrm{~K}$ & $T=20 \mathrm{~K}$ \\
\hline InAs/GaAsN QDs & $0.79 \mathrm{~V}$ & $1.03 \mathrm{~V}$ & $1.15 \mathrm{~V}$ & $1.23 \mathrm{~V}$ \\
\hline InAs/GaAs QDs & $0.99 \mathrm{~V}$ & $1.22 \mathrm{~V}$ & $1.43 \mathrm{~V}$ & $1.51 \mathrm{~V}$ \\
\hline GaAs reference & $1.00 \mathrm{~V}$ & $1.28 \mathrm{~V}$ & $1.41 \mathrm{~V}$ & $1.48 \mathrm{~V}$ \\
\hline
\end{tabular}

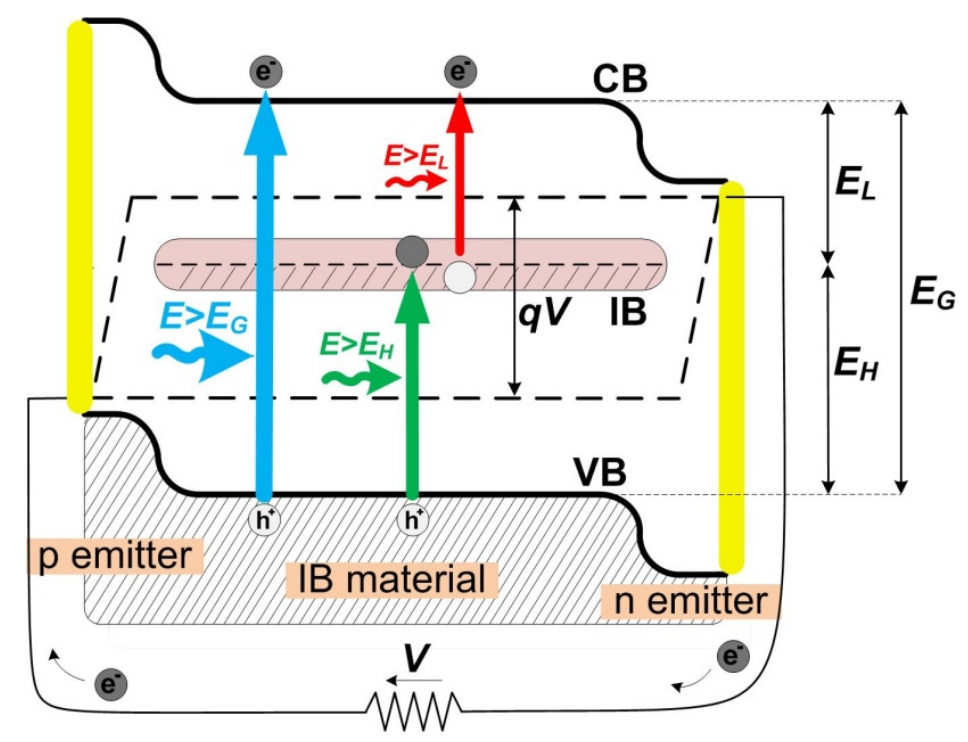

Figure 1. Band diagram of an IBSC where the IB material is shown hosting a three-band system. The three existing sub-bandgaps are labeled as $E_{\mathrm{G}}, E_{\mathrm{H}}$ and $E_{\mathrm{L}}$ and the associated electronic transitions are indicated with arrows. 

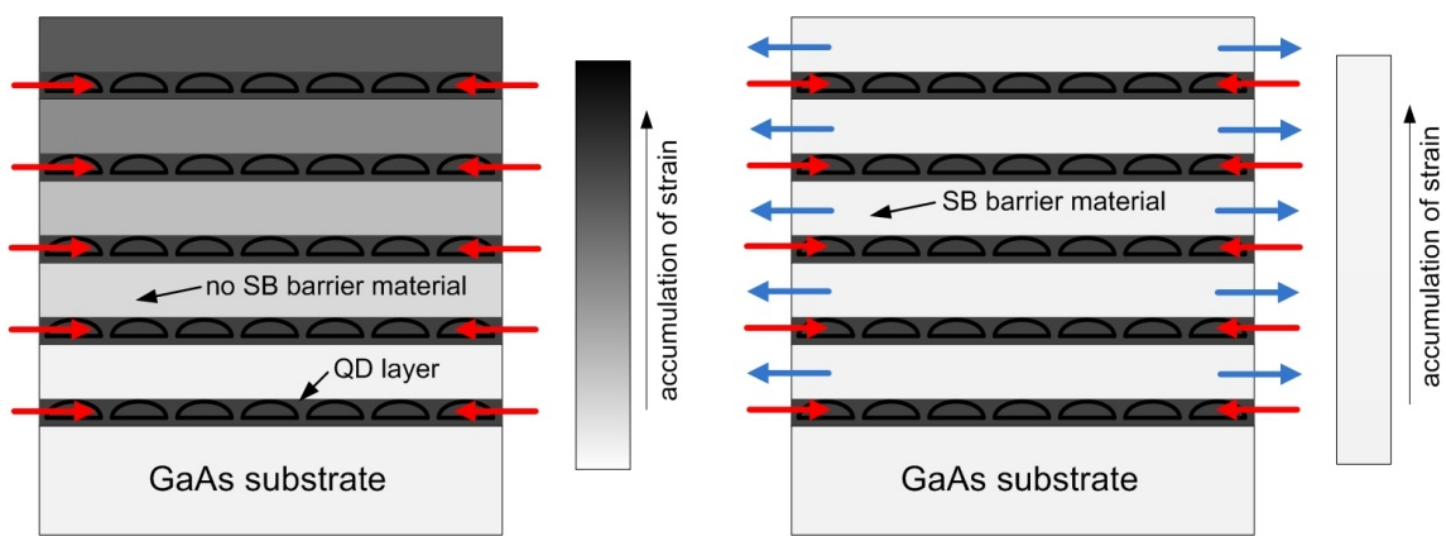

Figure 2. Sketch of the stacked InAs QD layers on GaAs substrate with (right) and without (left) strain compensation in the barrier. A bar on the right of each structure indicates the strain accumulated throughout the vertical axis.

\begin{tabular}{|c|c|}
\hline Ti/Pt/Au & \\
\hline $50 \mathrm{~nm}, p^{+}-\mathrm{GaAs}\left(510^{18} \mathrm{~cm}^{-3}\right)$ & contact \\
\hline $30 \mathrm{~nm}, \mathrm{p}^{+}-\mathrm{Al}_{0.4} \mathrm{Ga}_{0.6} \mathrm{As}$ & window \\
\hline $250 \mathrm{~nm}, p$ - GaAs $\left(210^{18} \mathrm{~cm}^{-3}\right)$ & p-emitter \\
\hline $20 \mathrm{~nm}, i-\mathrm{GaN}_{0.01} \mathrm{As}_{0.99}$ & \multirow{2}{*}{$\begin{array}{c}\text { X25 QD layers } \\
(500 \mathrm{~nm})\end{array}$} \\
\hline InAs, $2.0 \mathrm{ML}$ & \\
\hline \multicolumn{2}{|l|}{$20 \mathrm{~nm}, i-\mathrm{GaN}_{0.01} \mathrm{As}_{0.99}$} \\
\hline $1,000 \mathrm{~nm}, n-\mathrm{GaAs}\left(110^{17} \mathrm{~cm}^{-3}\right)$ & $n$-emitter \\
\hline $250 \mathrm{~nm}, n^{+}-$GaAs $\left(110^{18} \mathrm{~cm}^{-3}\right)$ & buffer \\
\hline Substrate, $n^{+}$-GaAs (001) & \\
\hline AuGe/Ni/Au & \\
\hline
\end{tabular}

Figure 3. Sketch of the layer structure of the InAs/GaAsN QD cell. 


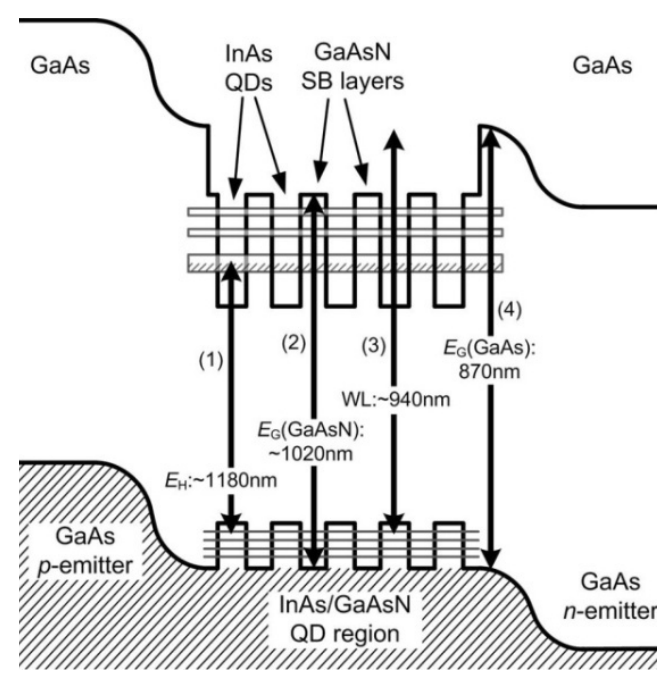

(a)

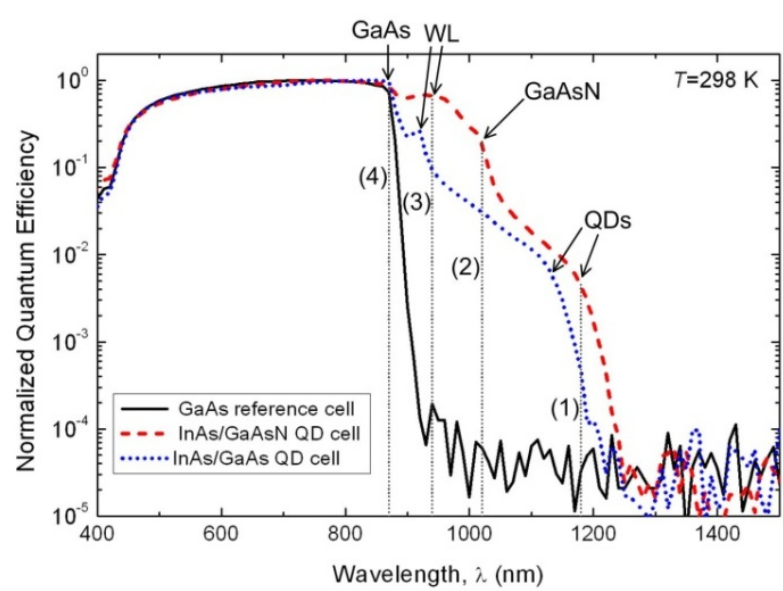

(b)

Figure 4. (a) Sketch of the band diagram of an InAs/GaAsN QD cell. (b) Normalized quantum efficiency of InAs/GaAsN QD cell compared to InAs/GaAs QD cell and GaAs reference cell. The same transition energies are identified in both graphs and represented in bracketed numbers.



Figure 5. Concentrated light $J_{\mathrm{L}}-V_{\mathrm{OC}}$ and dark $J-V$ characteristics of the $\operatorname{InAs} / \mathrm{GaAs}_{0.99} \mathrm{~N}_{0.01}$ QD cell at different temperatures. A vertical line at $1.22 \mathrm{eV}$ represents $E_{\mathrm{G}}\left(\mathrm{GaAs}_{0.99} \mathrm{~N}_{0.01}\right)$. 